\title{
Progress of site survey for large solar telescopes in western China
}

\author{
Yu Liu ${ }^{1}$, Tengfei Song ${ }^{1}$, Xuefei Zhang ${ }^{1}$, Shunqing Liu ${ }^{1}$, Mingyu \\ Zhao $^{1}$, Zhanjun Tian ${ }^{1}$, Yuhu Miao ${ }^{1}$, Hongbo Li ${ }^{1}$, Jing Huang ${ }^{1}$, Baoyu \\ $\mathrm{Su}^{1}$, Yongyin $\mathrm{Lu}^{1}$, Xiaobo $\mathrm{Li}^{1}$ and Qiwu Song ${ }^{2}$ \\ ${ }^{1}$ Yunnan Observatories, Chinese Academy of Sciences, Kunming 650011, China \\ email: lyu@ynao.ac.cn \\ ${ }^{2}$ Purple Mountain Observatory, Chinese Academy of Sciences, Nanjing 210008, China \\ email: songqw@pmo.ac.cn
}

\begin{abstract}
Excellent sites are necessary for developing and installing ground-based large telescopes. For very-high-resolution solar observations, it had been unclear whether there exist good candidate sites in the west areas in China, including the Tibetan Plateau and the Pamirs Plateau. The project of solar site survey for the next-generation large solar telescopes, i.e., the Chinese Giant Solar Telescope (CGST) and the large coronagraph, has been launched since 2011. Based on the close collaboration among Chinese solar society and the scientists from NSO, HAO and other institutes, we have successfully developed the standard instruments for solar site survey and applied them to more than 50 different sites distributed in Xinjiang, Tibet, Qinghai, Sichuan, Yunnan and Ningxia provinces. We have built two long-term monitoring sites in Tibet and the large Shangri-La to take systematic site data. Clear evidence, including the key parameters of seeing factor, sky brightness and water vapor content, has indicated that a few potential sites in the large Tibetan areas should obtain the excellent astronomical conditions for our purpose to develop CGST and large coronagraph. We introduce the fresh site survey results in this report.
\end{abstract}

Keywords. Site testing, surveys, Sun.

\section{Introduction}

The site survey is focused to look for the best candidate sites which should satisfy the strict requirement for a series of key parameters including average seeing factor $\mathrm{r}$, sky brightness, number of clear-sky days, precipitable water vapor content (PWVC), atmospheric coherence time, isoplanatic angle, meteorological information et al. The purpose of the site survey is expected to be accomplished as early as possible in recent years. The members of our site survey team come from a few institutes and universities including YNAO, PMO, NAOC, NJU and USTC.

The instruments for our survey mainly contain solar differential image motion monitor (S-DIMM), sky brightness monitor (SBM), PWVC monitor, DIMM, atmospheric temperature fluctuation monitor, and robot weather station, et al. Calibration for those instruments had been done carefully.

The schedule is roughly to be (1) In 2010, based on the study of meteorological and geographic information, we determine 10-20 candidate sites preliminarily. (2) During 2011-2012, we complete the site survey for the 10-20 candidate points. (3) During 20132014, we complete on-site field survey for 2 yr. (4) During 2015-2016, we finally determine the best sites for infrared and optical observation. 


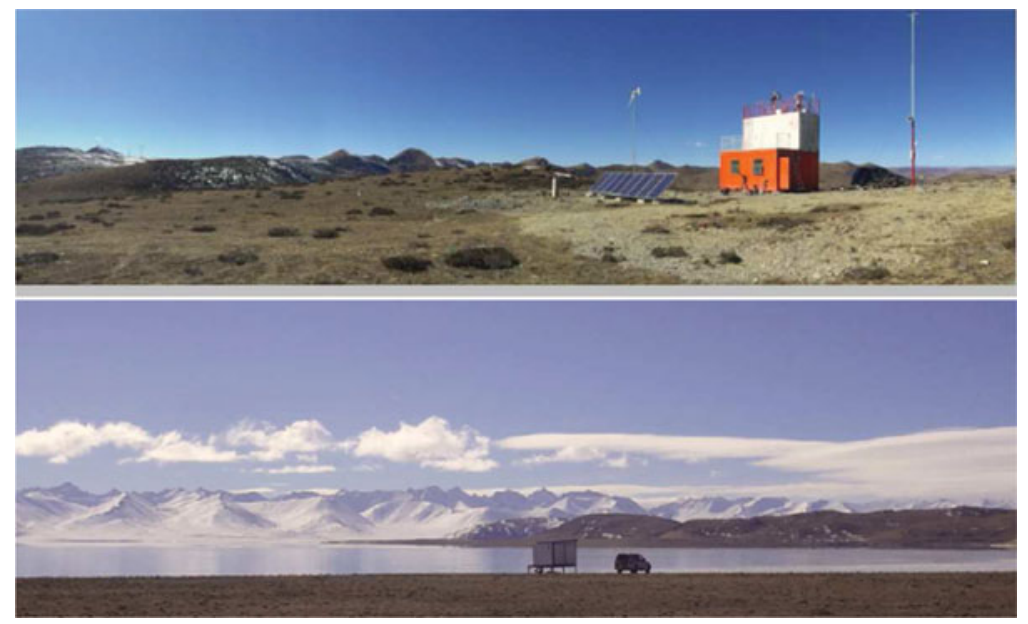

Figure 1. Top: The WMS (Daocheng) monitoring station located in western Sichuan. Buttom: The Namco monitoring station located in Tibet.
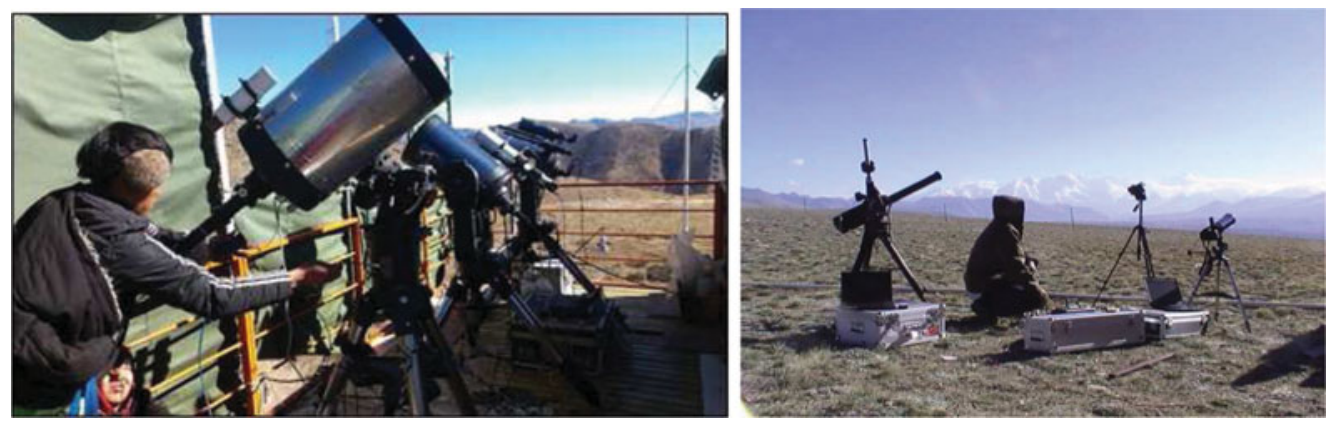

Figure 2. Professional site survey instruments after critical calibrations are used for the solar site survey.

\section{Strategy: remote and on-the-spot survey}

By using long-term meteorological data and GIS information, we can quickly decide and select the first-step candidate regions which are located in some areas of Pamier, Tibet and large Shargri-La in western China. The on-the-spot survey work has supplied us with the short-term data for the first comparison. Figure 1 shows the two potential sites, located in western Sichuan and Tibet, for long-term monitoring research. Figure 2 shows the instruments that are used for the site survey by the observers trained.

\section{Fixed-point monitoring}

Two best candidate sites, Namco lake and Wumingshan (WMS) mountain, have been chosen to make the long-term monitoring with SDIMM and the other instruments (Figure 1 and 2). Both the sites are located about $4700 \mathrm{~m}$ above the sea level. The average wind speeds for them are estimated less than $5 \mathrm{~m} / \mathrm{s}$ from the meteorological stations nearby.

As an example of the data, in Figure 3 the left frame shows the daytime seeing factor evolution, while the right one shows the sky brightness data during the whole day time. The average daytime seeing for both the two sites are more than $10 \mathrm{~cm}$ for the first year measurement. The sky brightness level at WMS monitoring station is excellent since the altitude there is so high that the atmosphere should be clean. A detailed report has been 

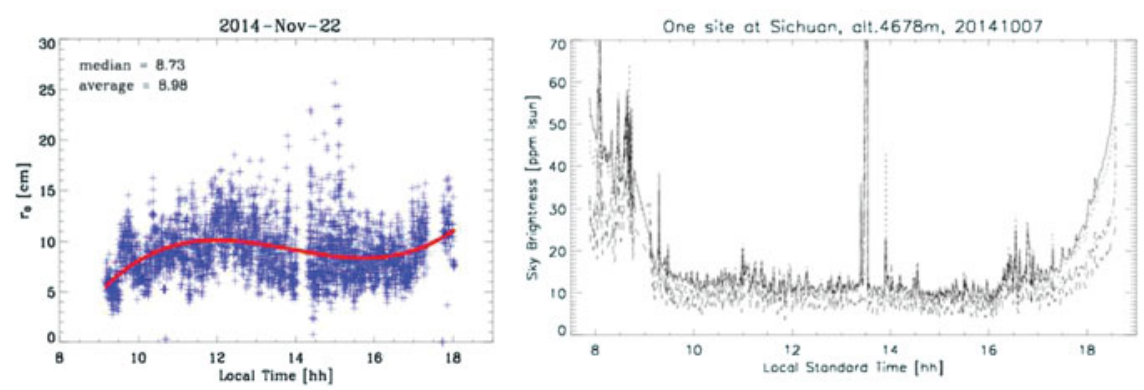

Figure 3. Left: Sample of seeing during day time. Right: Sample of sky brightness during day time.

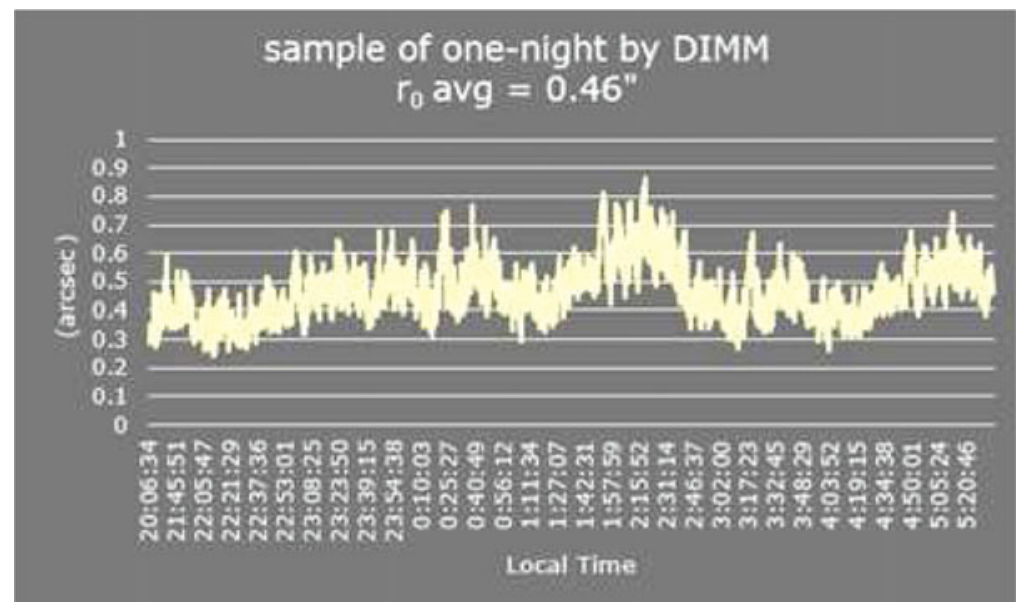

Figure 4. Sample of seeing during night time based on DIMM measurement.

completed recently for the site at WMS based on one-year monitoring measurements for both day and night seeing and the other parameters, which will be published elsewhere.

\section{Conclusion}

Based on the current results, the sites at Namco and WMS have been paid more and more attention for their excellent aspects in daytime seeing factor. Moreover, WMS owns better quality in clean atmosphere, excellent seeing factors for both daytime and night-time and number of yearly clear-sky days/nights.

Therefore, for WMS station, a new monitoring platform will be constructed at a location to fully escape from evening light pollution from village, road and airport. The day-and-night continuous monitoring at WMS will be kept at least for the next few years.

\section{References}

Ehgamberdiev, S., Baijumanov, A., Ilyasov, S., Sarazin, M., Tillayev, Y., Tokovinin, A., \& Ziad, A. 2000, Astron. Astrophys. Suppl. Ser., 145, 293

Lawrence, J., Ashley, M., Tokovinin, A., \& Travouillon, T. 2004, Nature, 431, 278

Liu, Y., Shen, Y., Zhang, X., \& Liu, N. 2012, Solar Phys., 279, 561

Liu, Y. \& Zhao, L. 2013, MNRAS, 434, 1674

Stone, R. 2010, Science, 329, 1002

Wu, N., Liu, Y., \& Zhao, M. 2015, Acta Astronomica Sinica, 56, 77

Zhao, M., Liu, Y., Elmhamdi, A., Kordi, A., \& Al-trabulsy, H., et al. 2014, MNRAS, 443, 1955 